Abanico Veterinario. Enero-Diciembre 2021; 11:1-12. http://dx.doi.org/10.21929/abavet2021.9

Artículo Original. Recibido: 21/02/2020. Aceptado: 20/01/2021. Publicado: 10/02/2021. Clave:2020-50.

\title{
Modificación de la fermentación ruminal in vitro para mitigación de metano mediante la adición de aceites esenciales de plantas y compuestos terpenoides
}

\author{
Modification of ruminal fermentation in vitro for methane mitigation by adding essential \\ oils from plants and terpenoid compounds
}

\section{Lucía Delgadillo-Ruiz $^{1 * I D}$, Rómulo Bañuelos-Valenzuela ${ }^{2 * \star ~ I D, ~ P e r l a ~ G a l l e g o s-~}$ Flores $^{1 \text { ID }}$, Francisco Echavarría-Cháirez ${ }^{3}$ ID , Carlos Meza-López ${ }^{2}$ ID , Norma Gaytán- Saldaña ${ }^{1}$ ID}

1 Unidad Académica de Ciencias Biológicas, Universidad Autónoma de Zacatecas. Avenida preparatoria s/n colonia Hidráulica, CP. 98068, Zacatecas, Zacatecas, México. ${ }^{2}$ Unidad Académica de Medicina Veterinaria y Zootecnia, Universidad Autónoma de Zacatecas. ${ }^{3}$ Instituto Nacional de Investigaciones Forestales, Agrícolas y Pecuarias. Campo experimental Zacatecas, México. *Autor responsable: Lucía Delgadillo-Ruiz. **Autor de correspondencia: Rómulo Bañuelos-Valenzuela. Carretera Panamericana Fresnillo-Zacatecas s/n, Centro, CP. 98500 Víctor Rosales, Zacatecas, México. luciadelgadillo@uaz.edu.mx, apozolero@hotmail.com, perla_gf17@hotmail.com, fechava1@yahoo.com, carmezlop@yahoo.com.mx, gaytanangelica1@gmail.com

\section{RESUMEN}

Los aceites esenciales de plantas son compuestos aromáticos volátiles, principalmente terpenoides, fenilpropanoides; monoterpenos, sesquiterpernos y alcoholes, estos presentan una amplia gama de actividad antimicrobiana y antioxidante, por lo que la adición de aceites esenciales de clavo, eucalipto, menta, romero, orégano y canela pueden modificar la fermentación ruminal al disminuir la población de bacterias productoras de metano y así tener una reducción de la producción de este gas. El objetivo fue evaluar diferentes aceites esenciales y compuestos terpenoides para mejorar la fermentación ruminal y la producción de ácidos grasos volátiles, atenuando la generación de metano. Se determinó la composición química (terpenoides) de los aceites, así como ácidos grasos volátiles (AGVs) por cromatografía de gases. Para la digestibilidad in vitro, se empleó la técnica de producción de gas in vitro y se utilizó líquido ruminal. El metano se infirió a partir de la concentración de AGVs. Se encontró que todos los aceites esenciales presentaron cada uno de los terpenoides en diferentes concentraciones, reportando la mayor concentración de carvacrol en el aceite esencial de clavo (303 $\left.\mathrm{mg} \mathrm{mL}^{-1}\right)$ y en orégano (1.20 mg mL-1); el terpineno se presentó en mayor cantidad en el aceite esencial de menta (4.83 $\left.\mathrm{mg} \mathrm{mL}^{-1}\right)$; para el aceite de menta y romero, linalol fue más elevado y para el limoneno la mayor concentración fue en el aceite de eucalipto (449 $\mathrm{mg} \mathrm{mL}^{-1}$ ) y romero $\left(12.42 \mathrm{mg} \mathrm{mL}^{-1}\right.$ ). Para la producción de gas en las digestibilidades el aceite esencial de eucalipto a dosis de 0.3 presentó $176 \mathrm{~mL} \mathrm{~g}^{-1} \mathrm{MS}$. Para digestibilidad in vitro el aceite de romero en dosis alta $(0.6 \mathrm{~mL}$ ), presentó la mejor fermentación ruminal ya que tuvo mejor mitigación de metano (716.83 $\mathrm{mM} / \mathrm{L}$ ) sin afectar de manera negativa la concentración de AGVs (acetato, 1892.2; propiónico, 526.14; butírico, $24.99 \mathrm{mM} / \mathrm{L}$ ), así como los terpenoides timol, linalol y limoneno en dosis alta. Se concluye que la mejor fermentación ruminal in vitro con mitigación de metano se observó con el aceite de romero y para los compuestos terpenoides fueron timol, linalol y limoneno en la dosis alta.

Palabras clave: Plantas, Compuestos terpenoides, Ácidos grasos volátiles y Metano.

\section{ABSTRACT}

Essential oils from plants are volatile aromatic compounds, mainly terpenoids, phenylpropanoids; monoterpenes, sesquiterpenes, and alcohols, these present a wide range of antimicrobial and antioxidant activity, so the addition of essential oils of clove, eucalyptus, mint, rosemary, oregano, and cinnamon can 
modify ruminal fermentation by reducing the population of bacteria producing methane and thus have a reduction in the production of this gas. The objective of this work was to evaluate different essential oils and terpenoid compounds to improve ruminal fermentation and the production of volatile fatty acids, attenuating the generation of methane. The chemical composition (terpenoids) of the oils, as well as volatile fatty acids (VFAs), were determined by gas chromatography. For in vitro digestibility, the in vitro gas production technique was used, and the ruminal liquid was used. Methane was inferred from the concentration of VFAs. It was found that all the essential oils presented each one of the terpenoids in different concentrations, reporting the highest concentration of carvacrol in the essential oil of clove (303 mg mL-1) and oregano $(1.20 \mathrm{mg} \mathrm{mL}-1)$; terpinene was presented in greater quantity in the essential oil of peppermint $(4.83 \mathrm{mg} \mathrm{mL}-$ 1); for peppermint and rosemary oil, linalool was higher and for limonene, the highest concentration was in eucalyptus oil (449 mg mL-1) and rosemary (12.42 mg mL-1). For gas production in digestibility, eucalyptus essential oil at a dose of 0.3 presented $176 \mathrm{~mL} \mathrm{g-1} \mathrm{DM.} \mathrm{For} \mathrm{in} \mathrm{vitro} \mathrm{digestibility,} \mathrm{rosemary} \mathrm{oil} \mathrm{in} \mathrm{high} \mathrm{dose}$ $(0.6 \mathrm{~mL})$ presented the best ruminal fermentation since it had better methane mitigation $(716.83 \mathrm{mM} / \mathrm{L})$ without negatively affecting the concentration of VFAs (acetate, 1892.2; propionic, 526.14; butyric, 24.99 $\mathrm{mM} / \mathrm{L}$ ), as well as the terpenoids thymol, linalool, and limonene in high doses. It is concluded that the best in vitro ruminal fermentation with methane mitigation was observed with rosemary oil and the terpenoid compounds were thymol, linalool, and limonene in the high dose.

Keywords: Plants, Terpenoid compounds, Volatile fatty acids, and Methane.

\section{INTRODUCCIÓN}

Las plantas aromáticas o arbustivas se han utilizado ampliamente de manera empírica en medicina tradicional para tratar diferentes padecimientos de salud (Cruz et al., 2017; Yuan et al., 2016), sin embargo, los efectos de éstas han generado interés en los sistemas de producción de ganado, ya que con la implementación de aditivos de plantas se puede modificar de manera efectiva la fermentación del rumen al inhibir la desaminación y metanogénesis que resulta en una reducción de metano $\left(\mathrm{CH}_{4}\right)$ entérico, $\mathrm{NH}_{3}-\mathrm{N}$ y acetato, y por lo tanto se producirá mayor concentración de propionato y butirato; así como disminución de $\mathrm{CH}_{4}$ entérico, el cual es un importante gas de efecto invernadero (Kurniawati et al., 2020; Wang et al., 2016; Kim et al., 2015).

Los aceites esenciales de plantas (AEP) son compuestos aromáticos volátiles, constituidos por una mezcla de metabolitos secundarios (MS); principalmente compuestos terpénicos, fenilpropanoides; monoterpenos, sesquiterpernos y alcoholes, aldehídos, éteres, esteres, cetonas y fenoles; que son principalmente responsables del aroma (Bakkali et al., 2008). Los AEP presentan una amplia gama de actividad antimicrobiana y antioxidante (Gallegos-Flores et al., 2019), por lo que han generado interés como alternativa de origen natural al uso de compuestos químicos para modificar la fermentación ruminal, ya que la implementación de aditivos sintéticos se ha visto limitada por la aparición de residuos en los productos de consumo humano, o por la resistencia que han generado ciertos microorganismos por el uso no terapéutico de antibióticos (ionóforos) en los rumiantes (Brown et al., 2017; Estévez y Cutuli, 2011). Algunos de los principales compuestos de AEP más comunes incluyen: timol y carvacrol (tomillo y orégano), eugenol (clavo), pineno (enebro), limoneno (eneldo), 1,8-cineole 
(eucalipto), cinamaldehído (canela), capsaicina (pimientos picantes), terpinene (árbol de té), alicina (ajo) y anethol (anís) (Kurniawati et al., 2020).

Existen plantas aromáticas que se han utilizado como especias comestibles, y a partir de las cuales sus aceites esenciales son ricos en compuestos terpenoides con una fuerte actividad antimicrobiana, los cuales pueden afectar el desarrollo y crecimiento de las bacterias ruminales e inhibir la metanogénesis; entre los aceites se encuentran: canela (Cinnamomum zeylanicum), clavo (Syzygium aromaticum), eupcalipto (Eucalyptus spp), menta (Mentha spicata), orégano (Origanum vulgare) y romero (Salvia rosmarinus) (Condo et al., 2018; Firmino et al., 2018; Dhakad et al., 2017).Dada la preocupación sobre la producción de gases efecto invernadero que contribuyen al calentamiento global, principalmente metano emitido por rumiantes, es necesario investigar el empleo de aceites esenciales, ya que al tener efecto antibacteriano se deduce que influyen en la microbiota del rumen, y por lo tanto modifican la fermentación y disminuyen la concentración de metano.

El objetivo de la presente investigación fue evaluar diferentes aceites esenciales y compuestos terpenoides, para incrementar la fermentación ruminal y la producción de ácidos grasos volátiles, atenuando la generación de metano.

\section{Material biológico}

\section{MATERIAL Y MÉTODOS}

Se utilizaron plantas de canela (Cinnamomum zeylanicum), clavo (Syzygium aromaticum), eucalipto (Eucalyptus spp), menta (Mentha spicata), orégano (Origanum vulgare) y romero (Salvia rosmarinus).

\section{Obtención de los aceites esenciales de plantas}

Las muestras de aceite esencial se obtuvieron a partir de la muestra seca por hidrodestilación durante $2 \mathrm{~h}$, utilizando un sistema Clevenger modificado. Durante el proceso de ebullición, el material seco absorbe el agua y el aceite esencial difunde a través de las paredes celulares por medio de ósmosis, luego es vaporizado y arrastrado por la corriente del evaporador (Teixeira et al., 2013).

\section{Composición química determinada por cromatografía de gases}

Se determinó mediante un cromatógrafo de gases (CG; Agilent Tecnologies serie 6890N), empleando la columna polar DB_WAXetr. Las condiciones de trabajo fueron; temperatura después de la inyección $250{ }^{\circ} \mathrm{C}$ a una presión de 12.13 psi con un flujo de He $36.5 \mathrm{~mL}$ $\mathrm{min}^{-1}$. Las condiciones para la columna fueron; temperatura inicial $50^{\circ} \mathrm{C}$ de cero a dos minutos, con un aumento de $10^{\circ} \mathrm{C}$ hasta llegar a $250^{\circ} \mathrm{C}$, utilizando un detector de flama ionizante (FID) a una temperatura de $210^{\circ} \mathrm{C}$ con un flujo de $\mathrm{H}_{2}$ de $40 \mathrm{~mL} \mathrm{~min}^{-1}$ y un flujo de aire de $450 \mathrm{~mL} \mathrm{~min}^{-1}$; previamente se realizó una curva de calibración. Los estándares utilizados fueron grado reactivo marca Sigma Aldrich: carvacrol, timol, limoneno, linalol y 
terpineno, con un porcentaje de pureza de $98,99.5,98,97$ y $85 \%$ respectivamente. Cada una de las determinaciones se realizó por triplicado Bañuelos et al. (2018).

\section{Preparación de compuestos terpenoides}

Los compuestos terpenoides utilizados para la digestibilidad in vitro, fueron los empleados como estándares en CG grado reactivo marca Sigma Aldrich: carvacrol, timol, limoneno, linalol y terpineno preparados con etanol al $50 \%$.

\section{Determinación de la producción de gas in vitro}

La alimentación de los ovinos para la producción de gas in vitro se utilizó fluido ruminal de dos ovinos de pelo, canulados y alimentados con una dieta que contenía $83 \%$ de heno ( $50 \%$ de alfalfa y $50 \%$ de paja de trigo) y $17 \%$ de concentrado (63\% maíz molido, $25 \%$ de harinolina, $5.5 \%$ de carbonato de calcio, $5.5 \%$ de fosfato mono-cálcico, $0.5 \%$ de pre mezcla de vitaminas $A, D$ y $E$ y $0.5 \%$ de microminerales). El alimento se proporcionó diariamente a las 08:00 y 16:00 horas con acceso libre al agua. Se alimentó a los ovinos por 30 días antes de la extracción del fluido ruminal, como tiempo de adaptación a la ración.

\section{Producción de gas in vitro}

Los aceites fueron adicionados de manera individual en cada una de las jarras de digestibilidad en diferente volumen (Ugbogu et al., 2019); como testigo se utilizó el sustrato de alfalfa sin la adición de aditivos. La producción de gas in vitro se determinó empleando el método propuesto por Theodorou et al. (1994); para lo cual se utilizaron unidades de fermentación (UF) de $120 \mathrm{~mL}$, para cada muestra. En el registro de gas producido, se utilizó un medidor de presión marca Sper Scientific. La presión de gas fue acumulativa y determinada en unidades de presión (Psi); el tiempo de medición fue a las $3,6,9,12,24$ y $48 \mathrm{~h}$; para cada volumen de los diferentes aceites, realizando tres repeticiones.

\section{Determinación de ácidos grasos volátiles en líquido ruminal}

Los AGVs (acético, propiónico y butírico) se cuantificaron por cromatografía de gases. Las condiciones de trabajo fueron; temperatura de entrada después de la inyección de la muestra es de $50^{\circ} \mathrm{C}$ a una presión de 12.13 psi con un flujo de He $36.5 \mathrm{~mL} \mathrm{~min}^{-1}$. Las condiciones para la columna fueron; temperatura inicial $50^{\circ} \mathrm{C}$, de cero a dos minutos con un aumento de $10^{\circ} \mathrm{C}$ por minuto hasta llegar a $250{ }^{\circ} \mathrm{C}$, manteniendo esta temperatura constante por 5 minutos, para luego descender a $50{ }^{\circ} \mathrm{C}$ manteniendo por dos minutos con un flujo de He de $1.6 \mathrm{~mL} \mathrm{~min}^{-1}$ a una presión de 12.13 psi y una velocidad promedio de $25 \mathrm{~cm} \mathrm{~s}^{-1}$. Se utilizó un detector de flama ionizante (FID) a una temperatura de $210^{\circ} \mathrm{C}$ con un flujo de $\mathrm{H}_{2}$ de $40 \mathrm{~mL} \mathrm{~min}^{-1} \mathrm{y}$ un flujo de aire de $450 \mathrm{~mL} \mathrm{~min}^{-1}$; previamente se realizó una curva de calibración. Los estándares utilizados fueron grado reactivo marca Sigma Aldrich: acético, propiónico y butírico, con un porcentaje de pureza de 99.5, 98 y 99\% respectivamente. Cada una de las determinaciones se realizó por triplicado. 


\section{Determinación de metano}

El metano se infirió a partir de la concentración de AGV, mediante la aplicación de modelos matemáticos no lineales establecido por Moss et al. (2000), donde se señala que la producción de $\mathrm{CH}_{4}$ se puede calcular de forma estequiométrica, empleando la siguiente ecuación:

$$
\left.\left.\left.\left[\mathrm{CH}_{4}\right]=0.45 C_{2} \text { (acetato }\right)-0.275 C_{3} \text { (propionato }\right)+0.4 C_{4} \text { (butirato }\right)
$$

\section{Análisis estadístico}

El análisis estadístico para la producción de gas, ácidos grasos volátiles y metano se realizó mediante un análisis de varianza, utilizando un diseño completamente al azar y la

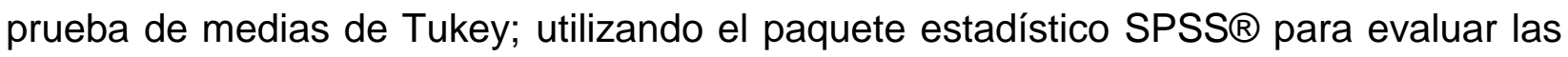
diferencias estadísticas $(p<0.05)$ (Cytel Software, 2010). La fuente de variación considerada fueron los aceites esenciales y compuestos terpenoides; para ácidos grasos volátiles se consideraron como variables: ácido acético, propiónico y butírico.

\section{RESULTADOS Y DISCUSIÓN}

Para los aceites esenciales de canela (Cinnamomum zeylanicum), clavo (Syzygium aromaticum), eucalipto (Eucalyptus spp), menta (Mentha spicata), orégano (Origanum vulgare) y romero (Salvia rosmarinus), se observó que la mayor concentración de carvacrol y timol está presente en los aceites de clavo (carvacrol $303 \mathrm{mg} \mathrm{mL}^{-1}$ ), y orégano (carvacrol, $1.652 \mathrm{mg} \mathrm{mL}^{-1}$; timol, $0.247 \mathrm{mg} \mathrm{mL}^{-1}$ ) (Cuadro 1). se ha reportado que estos dos compuestos tienen efecto antibacteriano contra bacterias gram negativas y positivas, para los cuales se conoce que el mecanismo de acción es incrustarse en la membrana de la célula bacteriana, provocando la desintegración de esta estructura, seguido de una lisis celular (Rodríguez-García et al., 2015; Friedman, 2014; Béjaoui et al., 2013; GarcíaGarcía et al., 2011); por lo que este efecto antibacteriano puede influir en la población de la microbiota ruminal y por lo tanto modificar la fermentación ruminal.

Cuadro 1. Compuestos terpenoides presentes en aceites esenciales analizados por cromatografía de gases

\begin{tabular}{cccccc}
\hline ACEITE & $\begin{array}{c}\text { Carvacrol } \\
\mathbf{m g ~ m L}^{-1}\end{array}$ & $\begin{array}{c}\text { Timol } \\
\mathbf{m g ~ m L}^{-1}\end{array}$ & $\begin{array}{c}\text { Linalol } \\
\mathbf{m g ~ m L}^{-1}\end{array}$ & $\begin{array}{c}\text { Terpineno } \\
\mathbf{m g ~ m L}^{-1}\end{array}$ & $\begin{array}{c}\text { Limoneno } \\
\mathbf{m g ~ m}^{-1}\end{array}$ \\
\hline Aceite esencial de canela & 0.0375 & 0.0108 & 0.047 & 0.1431 & 2.5167 \\
Aceite esencial de clavo & 303 & 0.0068 & 0.0383 & 0.2753 & 1.5496 \\
Aceite esencial de eucalipto & 0.07 & 0.0142 & 0.4621 & 0.8725 & 499 \\
Aceite esencial de menta & 0.0169 & 0.025 & 3.9401 & 4.8388 & 9.56 \\
Aceite esencial de orégano & 1.652 & 0.2474 & 0.0878 & 0 & 0.1449 \\
Aceite esencial de romero & 0.0524 & 0.0753 & 8.865 & 0.3725 & 12.425 \\
\hline
\end{tabular}

Nile et al. (2017) reportan que los aceites esenciales son ricos en terpenos (carvacrol, citral, linalol y geraniol); y compuestos fenólicos coincidiendo en el presente trabajo, ya que se encontraron ambos compuestos. Albado et al. (2001) reportó la presencia de 
compuestos terpenoides, fenoles y compuestos relacionados metabólicamente con el carvacrol en aceites esencial de orégano; por lo que esta investigación coincide con el presente estudio, ya que se identificaron los terpenoides en el aceite de orégano (carvacrol, timol y linalol), aunque en diferentes concentraciones. Bañuelos et al. (2018) menciona que los terpenoides constituyeron $11.2 \%$ del aceite con $\alpha$-pineno (1.3\%), limoneno (3\%) y 1,8-cineole (2.9\%), como los monoterpenos principales en el aceite esencial de orégano y $R$. graveolens. La presencia de limoneno en la presente investigación coincidió con esos resultados.

Los compuestos identificados son importantes por su actividad farmacológica; por ejemplo, el limoneno es antibacteriano, antifúngico, antiséptico y antiviral; el timol es antibacteriano, antifúngico, antiinflamatorio, antioxidante, antirreumático y antiséptico; el carvacrol es antibacteriano, antifúngico, antiinflamatorio, antiséptico, antiespasmódico y expectorante (Sorentino y Landmesser, 2005).

En la técnica de digestibilidad in vitro, la mayor obtención de gas en la técnica de digestibilidad in vitro (cuadro 2) se observó en el aceite esencial de eucalipto en sus tres dosis $\left(0.1=157.59 \pm 3.62 \mathrm{~mL} \mathrm{~g}^{-1} \mathrm{MS}, 0.3=176.86 \pm 1.10 \mathrm{~mL} \mathrm{~g}^{-1} \mathrm{MS}\right.$ y $0.6=175.30 \pm 3.62 \mathrm{~mL}$ $\mathrm{g}^{-1} \mathrm{MS}$ ), Wang et al. (2016) reportaron que al usar extractos de plantas medicinales no siempre existe una tendencia de aumentar la concentración de gas ruminal ( $\mathrm{mL} \mathrm{g}^{-1} \mathrm{MS}$ ); ya que algunos de ellos pueden tener el efecto contrario.

En los compuestos terpenoides se observa una baja producción de gas para la dosis de $0.6 \mathrm{~mL}$, registrando $48.24 \pm 3.41,29.77 \pm 4.87,28.63 \pm 0.50,28.98 \pm 3.09$ y $29.80 \pm 3.12 \mathrm{~mL} \mathrm{~g}^{-}$ ${ }^{1} \mathrm{MS} \pm \mathrm{DE}$ (timol, carvacrol, linalol, limoneno y terpineno respectivamente). Chouhan et al. (2017) y Gallegos-Flores et al. (2019) reportan que los metabolitos secundarios (terpenoides) de plantas, son reconocidos como agentes antimicrobianos que actúan contra las bacterias, protozoos y hongos; por lo que este efecto se ve reflejado en la poca producción de gas, debido a que inhibe el crecimiento de las bacterias metanogénicas ruminales, y por lo tanto los ácidos acético, propiónico y butírico son los que se generan en mayor cantidad durante la fermentación de los sustratos en el rumen.

La concentración de gas (total), AGV's y $\mathrm{CH}_{4}$ se presenta en el (cuadro 3); la producción de ácido propiónico se inhibió completamente en los aceites esenciales de eucalipto y orégano (dosis 0.1 ); mientras que en los compuestos terpenoides la producción de gas se inhibió en timol, dosis 0.3 ; carvacrol 0.6 ; linalol 0.3 ; limoneno 0.1 y 0.3 y terpineno 0.1 ; a excepción de aceite esencial de romero (dosis 0.1 ). Todas las dosis disminuyeron la producción de ácido propiónico en comparación con el testigo de alfalfa. La producción de ácido butírico se inhibió en el aceite esencial de clavo y canela en dosis de 0.1 y 0.3 ; mientras que la mayor producción de butírico se presentó en limoneno a la dosis de 0.1 (684.93 $\pm 0.09 \mathrm{mM} / \mathrm{L} \pm \mathrm{DE}$ ), pero se inhibe la producción de acético y propiónico.

Sejian et al. (2015) señalan que del 40 al $60 \%$ del total de los gases de efecto invernadero (GEI) de la ganadería provienen de la fermentación entérica, el manejo del estiércol y las diferentes actividades relacionadas con la obtención de alimentos para los animales. Por lo que los compuestos terpenoides presentan disminución de metano; tal es el caso del 
limoneno en su dosis 0.1 , presentando alta producción de ácido butírico, pero inhibición de acético y propiónico.

Cuadro 2. Producción de gas con las diferentes dosis de los aceites y terpenoides

\begin{tabular}{|c|c|c|c|c|c|c|c|}
\hline \multirow[b]{2}{*}{ Muestra } & \multirow[b]{2}{*}{$\begin{array}{c}\text { Dosis } \\
\text { (mL) }\end{array}$} & \multicolumn{6}{|c|}{ Producción de gas total en $\mathrm{mL} \mathrm{g}^{-1} \mathrm{MS} \pm \mathrm{DE}$} \\
\hline & & $3 \mathrm{~h}$ & $6 \mathrm{~h}$ & $9 \mathrm{~h}$ & $12 \mathrm{~h}$ & $24 \mathrm{~h}$ & $48 \mathrm{~h}$ \\
\hline & 0.1 & $11.67 \pm 0.14$ & $28.52 \pm 0.46$ & $51.13 \pm 1.44$ & $75.38 \pm 2.29$ & $108.57 \pm 0.16$ & $144.94 \pm 1.44$ \\
\hline \multirow[t]{3}{*}{ Aceite esencial de romero } & 0.3 & $11.87 \pm 1.85$ & $27.87 \pm 3.56$ & $49.09 \pm 6.15$ & $72.13 \pm 9.32$ & $108.80 \pm 10.17$ & $146.98 \pm 5.55$ \\
\hline & 0.6 & $9.26 \pm 0.04$ & $22.84 \pm 6.01$ & $40.39 \pm 15.97$ & $58.95 \pm 28.24$ & $94.41 \pm 10.17$ & $139.13 \pm 3.73$ \\
\hline & 0.1 & $6.39 \pm 5.12$ & $11.42 \pm 5.90$ & $17.91 \pm 1.07$ & $22.64 \pm 1.35$ & $28.22 \pm 10.49$ & $35.76 \pm 6.15$ \\
\hline \multirow[t]{3}{*}{ Aceite esencial de clavo } & 0.3 & $9.15 \pm 1.96$ & $15.39 \pm 2.81$ & $19.42 \pm 1.07$ & $20.72 \pm 1.35$ & $21.58 \pm 4.69$ & $22.48 \pm 9.39$ \\
\hline & 0.6 & $9.31 \pm 0.11$ & $14.34 \pm 0.75$ & $17.81 \pm 1.14$ & $19.01 \pm 1.21$ & $19.77 \pm 1.28$ & $20.72 \pm 1.24$ \\
\hline & 0.1 & $13.63 \pm 2.31$ & $33.90 \pm 4.23$ & $61.06 \pm 6.26$ & $88.38 \pm 7.61$ & $122.18 \pm 10.49$ & $157.59 \pm 3.62$ \\
\hline \multirow[t]{3}{*}{ Aceite esencial de eucalipto } & 0.3 & $16.90 \pm 0.96$ & $39.89 \pm 2.28$ & $69.92 \pm 3.98$ & $99.14 \pm 4.45$ & $137.02 \pm 3.09$ & $176.86 \pm 1.10$ \\
\hline & 0.6 & $15.54 \pm 5.69$ & $36.67 \pm 7.86$ & $64.28 \pm 5.39$ & $92.85 \pm 4.45$ & $132.64 \pm 13.00$ & $175.30 \pm 3.62$ \\
\hline & 0.1 & $8.50 \pm 3.45$ & $18.01 \pm 2.19$ & $27.26 \pm 0.59$ & $33.50 \pm 3.72$ & $45.97 \pm 11.79$ & $65.29 \pm 4.49$ \\
\hline \multirow[t]{3}{*}{ Aceite esencial de menta } & 0.3 & $9.26 \pm 0.53$ & $14.64 \pm 2.38$ & $20.93 \pm 4.48$ & $23.29 \pm 7.22$ & $24.85 \pm 4.94$ & $26.26 \pm 7.60$ \\
\hline & 0.6 & $7.49 \pm 1.24$ & $11.42 \pm 2.28$ & $14.24 \pm 4.73$ & $15.39 \pm 5.58$ & $16.20 \pm 6.12$ & $16.80 \pm 6.69$ \\
\hline & 0.1 & $13.38 \pm 0.71$ & $21.10 \pm 0.82$ & $26.43 \pm 0.92$ & $28.24 \pm 0.84$ & $29.30 \pm 0.78$ & $30.66 \pm 0.82$ \\
\hline \multirow[t]{3}{*}{ Aceite esencial de orégano } & 0.3 & $14.39 \pm 1.64$ & $22.26 \pm 2.40$ & $27.74 \pm 2.60$ & $29.43 \pm 2.38$ & $30.41 \pm 2.15$ & $31.82 \pm 1.96$ \\
\hline & 0.6 & $12.07 \pm 2.28$ & $18.86 \pm 3.79$ & $24.07 \pm 4.41$ & $26.06 \pm 4.52$ & $27.36 \pm 4.48$ & $29.05 \pm 4.68$ \\
\hline & 0.1 & $14.11 \pm 3.22$ & $24.47 \pm 8.41$ & $31.71 \pm 2.72$ & $34.58 \pm 37.90$ & $35.91 \pm 3.68$ & $37.88 \pm 9.66$ \\
\hline \multirow[t]{3}{*}{ Aceite esencial de canela } & 0.3 & $14.18 \pm 0.05$ & $22.01 \pm 1.74$ & $27.87 \pm 2.72$ & $29.83 \pm 3.36$ & $30.73 \pm 3.66$ & $32.39 \pm 3.88$ \\
\hline & 0.6 & $15.29 \pm 0.78$ & $24.22 \pm 1.56$ & $30.31 \pm 1.73$ & $32.44 \pm 1.85$ & $33.70 \pm 2.10$ & $35.66 \pm 2.31$ \\
\hline & 0.1 & $1.95 \pm 0.37$ & $5.03 \pm 0.53$ & $10.04 \pm 1.20$ & $33.74 \pm 4.08$ & $41.26 \pm 4.49$ & $47.99 \pm 4.92$ \\
\hline \multirow[t]{3}{*}{ Timol } & 0.3 & $2.34 \pm 0.04$ & $5.37 \pm 0.06$ & $9.89 \pm 0.46$ & $31.84 \pm 2.53$ & $39.17 \pm 3.13$ & $45.83 \pm 3.55$ \\
\hline & 0.6 & $2.14 \pm 0.14$ & $4.79 \pm 0.70$ & $9.28 \pm 1.16$ & $31.82 \pm 3.22$ & $40.19 \pm 3.91$ & $48.24 \pm 3.41$ \\
\hline & 0.1 & $2.17 \pm 0.04$ & $5.01 \pm 0.12$ & $9.68 \pm 0.43$ & $14.97 \pm 0.46$ & $22.28 \pm 0.15$ & $28.47 \pm 0.38$ \\
\hline \multirow[t]{3}{*}{ Carvacrol } & 0.3 & $2.52 \pm 0.01$ & $5.38 \pm 0.04$ & $9.70 \pm 0.25$ & $14.86 \pm 0.38$ & $21.73 \pm 0.39$ & $29.06 \pm 0.85$ \\
\hline & 0.6 & $2.03 \pm 0.08$ & $4.72 \pm 0.51$ & $9.07 \pm 0.48$ & $13.90 \pm 1.21$ & $21.67 \pm 2.74$ & $29.77 \pm 4.87$ \\
\hline & 0.1 & $2.06 \pm 0.04$ & $5.61 \pm 0.04$ & $10.80 \pm 0.04$ & $16.31 \pm 0.04$ & $22.79 \pm 0.11$ & $30.37 \pm 1.90$ \\
\hline \multirow[t]{3}{*}{ Linalol } & 0.3 & $2.09 \pm 0.29$ & $5.47 \pm 0.33$ & $10.31 \pm 0.45$ & $16.54 \pm 1.89$ & $23.65 \pm 3.07$ & $31.98 \pm 1.86$ \\
\hline & 0.6 & $2.04 \pm 0.03$ & $5.27 \pm 0.14$ & $9.88 \pm 0.33$ & $15.05 \pm 0.46$ & $21.64 \pm 0.33$ & $28.63 \pm 0.50$ \\
\hline & 0.1 & $1.97 \pm 0.04$ & $5.35 \pm 0.03$ & $10.31 \pm 0.05$ & $15.63 \pm 0.06$ & $21.97 \pm 1.43$ & $29.18 \pm 0.09$ \\
\hline \multirow[t]{3}{*}{ Limoneno } & 0.3 & $2.16 \pm 0.02$ & $5.59 \pm 0.01$ & $10.48 \pm 0.00$ & $15.86 \pm 0.06$ & $22.36 \pm 0.01$ & $30.68 \pm 2.00$ \\
\hline & 0.6 & $1.95 \pm 0.02$ & $4.60 \pm 0.03$ & $8.70 \pm 0.27$ & $13.50 \pm 0.63$ & $20.10 \pm 0.82$ & $28.98 \pm 3.09$ \\
\hline & 0.1 & $2.02 \pm 0.06$ & $5.39 \pm 0.18$ & $10.19 \pm 0.31$ & $15.37 \pm 0.51$ & $21.41 \pm 0.07$ & $28.71 \pm 2.30$ \\
\hline \multirow[t]{2}{*}{ Terpineno } & 0.3 & $2.04 \pm 0.12$ & $5.22 \pm 0.11$ & $9.73 \pm 0.04$ & $14.60 \pm 0.03$ & $21.04 \pm 0.31$ & $28.92 \pm 1.67$ \\
\hline & 0.6 & $2.24 \pm 0.19$ & $4.90 \pm 0.28$ & $8.87 \pm 0.43$ & $13.48 \pm 0.69$ & $20.64 \pm 0.87$ & $29.80 \pm 3.12$ \\
\hline Alfalfa & 0 & $2.19 \pm 0.04$ & $4.96 \pm 0.41$ & $8.86 \pm 0.96$ & $13.53 \pm 0.92$ & $22.12 \pm 1.13$ & $32.30 \pm 5.41$ \\
\hline
\end{tabular}

* SM: materia seca. DE: Desviación estándar. 
Cuadro 3. Producción de gas total ( $\left.\mathrm{mL} \mathrm{g}^{-1} \mathrm{MS}\right)$, ácidos grasos volátiles ( $\left.\mathrm{mM} / \mathrm{L}\right)$ y metano in vitro en los diferentes aceites esenciales y terpenoides empleados

\begin{tabular}{|c|c|c|c|c|c|c|}
\hline \multirow[b]{2}{*}{ Muestra } & \multirow{2}{*}{$\begin{array}{l}\text { Dosis } \\
(\mathrm{mL})\end{array}$} & \multirow{2}{*}{$\begin{array}{c}\text { Producción total } \\
\text { de Gas (mL } \mathrm{g}^{-1} \\
\mathrm{MS}) \pm^{\star} \mathrm{DE}\end{array}$} & \multicolumn{3}{|c|}{ Ácidos Grasos Volátiles (mM/L) $\pm{ }^{\star} \mathrm{DE}$} & \multirow[b]{2}{*}{ Metano $\mathrm{mM} / \mathrm{L}$} \\
\hline & & & $\begin{array}{l}\text { Ácido } \\
\text { acético }\end{array}$ & $\begin{array}{c}\text { Ácido } \\
\text { Propiónico }\end{array}$ & $\begin{array}{c}\text { Ácido } \\
\text { butírico }\end{array}$ & \\
\hline \multirow{3}{*}{$\begin{array}{l}\text { Aceite esencial de } \\
\text { romero }\end{array}$} & 0.1 & $144.94 \pm 1.44^{\mathrm{a}}$ & $2380.5 \pm 0.02^{\mathrm{a}}$ & $782.20 \pm 0.15^{a}$ & $43.62 \pm 0.19^{b}$ & $873.57 \pm 0.06^{\mathrm{bc}}$ \\
\hline & 0.3 & $146.98 \pm 5.55^{a}$ & $2154.3 \pm 0.03^{a}$ & $664.61 \pm 0.16^{\mathrm{a}}$ & $32.88 \pm 0.19^{b}$ & $799.85 \pm 0.07^{\mathrm{bc}}$ \\
\hline & 0.6 & $139.13 \pm 3.73^{a}$ & $1892.2 \pm 0.04^{\mathrm{a}}$ & $526.14 \pm 0.16^{a}$ & $24.99 \pm 0.19^{b}$ & $716.83 \pm 0.07^{\mathrm{bc}}$ \\
\hline \multirow{3}{*}{$\begin{array}{c}\text { Aceite esencial de } \\
\text { clavo }\end{array}$} & 0.1 & $35.76 \pm 6.15^{c}$ & $435.87 \pm 0.13^{b}$ & $39.15 \pm 0.19^{b}$ & $0.00 \pm 0.00^{c}$ & $185.37 \pm 0.01^{\mathrm{a}}$ \\
\hline & 0.3 & $22.48 \pm 9.39^{c}$ & $312.6 \pm 0.13^{b}$ & $40.46 \pm 0.19^{b}$ & $0.00 \pm 0.00^{c}$ & $129.54 \pm 0.01^{\mathrm{a}}$ \\
\hline & 0.6 & $20.72 \pm 1.24^{\mathrm{cd}}$ & $322.25 \pm 0.13^{b}$ & $40.85 \pm 0.19^{b}$ & $15.16 \pm 0.19^{\mathrm{bc}}$ & $139.85 \pm 0.12^{\mathrm{a}}$ \\
\hline \multirow{3}{*}{$\begin{array}{l}\text { Aceite esencial de } \\
\text { eucalipto }\end{array}$} & 0.1 & $157.59 \pm 3.62^{\mathrm{a}}$ & $2343.2 \pm 0.02^{\mathrm{a}}$ & $0.00 \pm 0.00^{c}$ & $617.94 \pm 0.10^{\mathrm{a}}$ & $1301.62 \pm 0.07^{c}$ \\
\hline & 0.3 & $176.86 \pm 1.10^{\mathrm{a}}$ & $2291.8 \pm 0.02^{\mathrm{a}}$ & $676.56 \pm 0.16^{\mathrm{a}}$ & $37.97 \pm 0.19^{b}$ & $860.46 \pm 0.06^{\mathrm{bc}}$ \\
\hline & 0.6 & $175.30 \pm 3.62^{\mathrm{a}}$ & $2514.2 \pm 0.01^{\mathrm{a}}$ & $764.87 \pm 0.43^{a}$ & $46.61 \pm 0.19^{b}$ & $939.72 \pm 0.06^{b c}$ \\
\hline \multirow{3}{*}{$\begin{array}{l}\text { Aceite esencial de } \\
\text { menta }\end{array}$} & 0.1 & $65.29 \pm 4.49^{b}$ & $244.9 \pm 11.77^{\mathrm{b}}$ & $51.76 \pm 25.88^{a}$ & $18.39 \pm 3.82^{\mathrm{bc}}$ & $103.32 \pm 33.26^{a}$ \\
\hline & 0.3 & $26.26 \pm 7.60^{c}$ & $868.6 \pm 12.01^{a b}$ & $51.00 \pm 25.69^{a}$ & $106.3 \pm 44.19^{a b}$ & $419.42 \pm 33.55^{b}$ \\
\hline & 0.6 & $16.80 \pm 6.69^{d}$ & $234.92 \pm 4.36^{\mathrm{b}}$ & $52.84 \pm 0.19^{b}$ & $21.46 \pm 0.19^{b}$ & $99.77 \pm 2.74^{\mathrm{a}}$ \\
\hline \multirow{3}{*}{$\begin{array}{c}\text { Aceite esencial de } \\
\text { orégano }\end{array}$} & 0.1 & $30.66 \pm 0.82^{c}$ & $121.7 \pm 0.14^{b}$ & $0.00 \pm 0.00^{c}$ & $10.32 \pm 0.19^{\mathrm{bc}}$ & $58.89 \pm 0.20^{a}$ \\
\hline & 0.3 & $31.82 \pm 1.96^{c}$ & $117.0 \pm 0.15^{b}$ & $21.72 \pm 0.19^{b}$ & $17.95 \pm 0.19^{\mathrm{bc}}$ & $53.88 \pm 0.13^{a}$ \\
\hline & 0.6 & $29.05 \pm 4.68^{c}$ & $105.6 \pm 0.15^{b}$ & $25.85 \pm 0.19^{b}$ & $17.56 \pm 0.19^{b c}$ & $47.48 \pm 0.13^{a}$ \\
\hline \multirow{4}{*}{$\begin{array}{c}\text { Aceite esencial de } \\
\text { canela }\end{array}$} & 0.1 & $37.88 \pm 9.66^{b c}$ & $473.2 \pm 0.12^{b}$ & $108.35 \pm 0.19^{\mathrm{ab}}$ & $0.00 \pm 0.00^{c}$ & $183.15 \pm 0.01^{a}$ \\
\hline & 0.3 & $32.39 \pm 3.88^{c}$ & $249.48 \pm 0.14^{b}$ & $47.47 \pm 0.19^{b}$ & $0.00 \pm 0.00^{c}$ & $99.21 \pm 0.01^{\mathrm{a}}$ \\
\hline & 0.6 & $35.66 \pm 2.31^{c}$ & $323.43 \pm 0.13^{b}$ & $72.03 \pm 0.19^{b}$ & $39.63 \pm 0.19^{b}$ & $141.59 \pm 0.12^{\mathrm{a}}$ \\
\hline & 0.1 & $47.99 \pm 4.92^{\mathrm{bc}}$ & $1884.20 \pm 0.04^{a}$ & $720.35 \pm 0.15^{a}$ & $39.87 \pm 0.19^{b}$ & $665.74 \pm 0.08^{b}$ \\
\hline \multirow[t]{3}{*}{ Timol } & 0.3 & $45.83 \pm 3.55^{\mathrm{bc}}$ & $1874.53 \pm 0.24^{a}$ & $0.00 \pm 0.00^{c}$ & $551.46 \pm 0.11^{\mathrm{a}}$ & $1064.12 \pm 0.21^{c}$ \\
\hline & 0.6 & $48.24 \pm 3.41^{\mathrm{bc}}$ & $1824.96 \pm 0.05^{a}$ & $696.55 \pm 0.15^{a}$ & $34.59 \pm 0.19^{b}$ & $643.52 \pm 0.08^{b}$ \\
\hline & 0.1 & $28.47 \pm 0.38^{c}$ & $1933.54 \pm 0.23^{a}$ & $0.00 \pm 0.00^{c}$ & $603.55 \pm 0.10^{a}$ & $1111.51 \pm 0.20^{\circ}$ \\
\hline \multirow[t]{3}{*}{ Carvacrol } & 0.3 & $29.06 \pm 0.85^{c}$ & $1826.95 \pm 0.05^{a}$ & $724.29 \pm 0.15^{a}$ & $35.87 \pm 0.19^{b}$ & $637.30 \pm 0.08^{\mathrm{b}}$ \\
\hline & 0.6 & $29.77 \pm 4.87^{c}$ & $1436.98 \pm 0.01^{\mathrm{a}}$ & $0.00 \pm 0.00^{c}$ & $438.03 \pm 0.13^{a}$ & $821.85 \pm 0.08^{\text {bc }}$ \\
\hline & 0.1 & $30.37 \pm 1.90^{c}$ & $1868.50 \pm 0.05^{a}$ & $731.91 \pm 0.15^{\mathrm{a}}$ & $38.56 \pm 0.19^{b}$ & $654.98 \pm 0.08^{b}$ \\
\hline \multirow[t]{3}{*}{ Linalol } & 0.3 & $31.98 \pm 1.86^{c}$ & $1891.33 \pm 0.04^{a}$ & $0.00 \pm 0.00^{c}$ & $582.39 \pm 0.11^{a}$ & $1084.05 \pm 0.09^{c}$ \\
\hline & 0.6 & $28.63 \pm 0.50^{c}$ & $1908.58 \pm 0.04^{a}$ & $713.00 \pm 0.15^{a}$ & $33.15 \pm 0.19^{b}$ & $676.05 \pm 0.08^{b}$ \\
\hline & 0.1 & $29.18 \pm 0.09^{c}$ & $1990.96 \pm 0.02^{a}$ & $0.00 \pm 0.00 \mathrm{c}$ & $603.49 \pm 0.10^{a}$ & $1137.33 \pm 0.07 \mathrm{c}$ \\
\hline \multirow[t]{3}{*}{ Limoneno } & 0.3 & $30.68 \pm 2.00^{c}$ & $1909.62 \pm 0.04^{a}$ & $0.00 \pm 0.00^{c}$ & $567.93 \pm 0.11^{\mathrm{a}}$ & $1086.50 \pm 0.09^{c}$ \\
\hline & 0.6 & $28.98 \pm 3.09^{c}$ & $1755.33 \pm 0.05^{a}$ & $625.03 \pm 0.34^{a}$ & $35.26 \pm 0.19^{b}$ & $632.12 \pm 0.27^{b}$ \\
\hline & 0.1 & $28.71 \pm 2.30^{c}$ & $0.00 \pm 0.00^{c}$ & $0.00 \pm 0.00^{a}$ & $684.93 \pm 0.09^{a}$ & $273.97 \pm 0.05^{\mathrm{ab}}$ \\
\hline \multirow[t]{2}{*}{ Terpineno } & 0.3 & $28.92 \pm 1.67^{c}$ & $1990.91 \pm 0.04^{a}$ & $762.28 \pm 0.15^{\mathrm{a}}$ & $40.87 \pm 0.19^{b}$ & $702.63 \pm 0.07^{\mathrm{bc}}$ \\
\hline & 0.6 & $29.80 \pm 3.12^{c}$ & $1886.46 \pm 0.27^{a}$ & $0.00 \pm 0.00^{c}$ & $583.48 \pm 0.10^{\mathrm{a}}$ & $1082.30 \pm 0.23^{c}$ \\
\hline Alfalfa & 0 & $32.30 \pm 5.41^{\mathrm{c}}$ & $1673.52 \pm 0.06^{a}$ & $775.33 \pm 0.15^{a}$ & $43.50 \pm 0.19^{b}$ & $557.27 \pm 0.08^{b}$ \\
\hline
\end{tabular}

* DE: Desviación estándar, valores de medias con letras distintas en la misma columna difieren estadísticamente $(p<0.05)$. 


\section{CONCLUSIONES}

La mitigación de metano se observó con el aceite de romero en su dosis máxima en la fermentación ruminal in vitro; ya que presenta incremento de las concentraciones de AGV's (acético, propiónico y butírico). Los compuestos terpenoides con mejor fermentación ruminal in vitro fueron timol, linalol y limoneno en la dosis máxima. Se sugiere profundizar en el uso de aceites esenciales de plantas, porque pudieran ser una alternativa en la búsqueda de productos orgánicos con mayor sustentabilidad.

\section{LITERATURA CITADA}

ALBADO PE, Sáez FG, S. Grabiel AS. 2001. Composición química y actividad antibacteriana del aceite esencial del Origanum vulgare (orégano). Revista Medica Herediana. 12(1):16-19. ISSN:

1729-214X.

http://www.scielo.org.pe/pdf/rmh/v12n1/v12n1ao3.pdf

BAKKALI F, Averbeck S, Averbeck D, Idaomar M. 2008. Biological effects of essential oils-a review. Food and Chemical Toxicology. 46(2):446-475. https://doi.org/10.1016/j.fct.2007.09.106

BAÑUELOS VR, Delgadillo RL, Echavarría CF, Delgadillo RO, Meza LC. 2018. Composición química y FTIR de extractos etanólicos de Larrea tridentata, Origanum vulgare, Artemisa ludoviciana y Ruta graveolens. Agrociencia. 52(3): 309-321. ISSN 2521-9766. http://www.scielo.org.mx/pdf/agro/v52n3/2521-9766-agro-52-03-309.pdf

BEJAOUI A, Boulila A, Boussaid M. 2013. Chemical composition and biological activities of essential oils and solvent extracts of Origanum vulgare subps. Glandulosum Desf. From Tunisia. Journal of Medicinal Plants Research. 7 (32): 2429-2435. https://doi.org/10.5897/JMPR11.902

BROWN K, Uwiera RRE, Kalmokoff ML, Brooks SPJ, Inglis GD. 2017. Antimicrobial growth promoter use in livestock: a requirement to understand their modes of action to develop effective alternatives. International Journal Antimicrobiology Agents. 49(1):1224. https://doi.org/10.1016 / j.ijantimicag.2016.08.006

CHOUHAN S, Sharma K, Guleria S. 2017. Antimicrobial activity of some essential oilspresent status and future perspectives. Medicines. 4(3):58. https://doi.org/10.3390/medicines4030058 
CONDO C, Anacarso I, Sabia C, Iseppi R, Anfelli I, Forti L, Niederhäusern S, Bondi M, Messi P. 2018. Antimicrobial activity of spice essential oils and their effectiveness in mature biofilms of human pathogens. Natural Product Research. 34(4):567-574. https://doi.org/10.1080/14786419.2018.1490904

CRUZ MC, Diaz-Gómez M, Sook-Oh M. 2017. Use of traditional herbal medicine as an alternative in dental treatment in Mexican dentistry: A review. Pharmaceutical Biology. 55(1): 1992-1998. https://doi.org/10.1080/13880209.2017.1347188

CYTEL SOFTWARE. 2010. Statxact 9 with Cytel studio. Statistical software for exact nonparametric inference. User manual. Cytel Software, New York, USA. Pp. 1345.

DHAKAD AK, Pandey VV, Beg S, Rawat JM. 2017. Biological, medicinal and toxicological significance of Eucalyptus leaf essential oil: a review. Journal of the Science of Food and Agriculture. 98(3):833-848. https://doi.org/10.1002/jsfa.8600

ESTÉVEZ RRM, Cutuli SMT. 2011. Alternativas en promoción del crecimiento tras la prohibición de los antibióticos I: Modificadores metabólicos y modificadores inmunológicos. Información Veterinaria, Revista de la Organización Colegial Veterinaria Española. 04:18-23. ISSN 1130-5436. http://www.colvet.es/sites/default/files/201512/2011_04_informacion_veterinariaabril_2011.pdf

FIRMINO D, Cavalcante T, Gomes GA, Firmino N, Rosa L, Carvalho M, Catunda F. 2018. Antibacterial and Antibiofilm Activities of Cinnamomum Sp. Essential Oil and Cinnamaldehyde: Antimicrobial Activities. Scientific World Journal. 2018:1-9. https://doi.org/10.1155 / 2018/7405736

FRIEDMAN M. 2014. Chemistry and Multibeneficial Bioactivities of Carvacrol (4Isopropyl-2-methylphenol), a Component of Essential Oils Produced by Aromatic Plants and Spices: Review. Journal of Agricultural and Food Chemistry. 62, 7652-7670. https://doi.org/10.1021/jf5023862|J

GALLEGOS-FLORES PI, Bañuelos-Valenzuela R, Delgadillo-Ruiz L, Meza-López C, Echavarría-Cháirez F. 2019. Actividad antibacteriana de cinco compuestos terpenoides: carvacrol, limoneno, linalool, a-terpineno y timol. Tropical and Subtropical $\begin{array}{llll}\text { Agroecosystems. } & \text { 22(2):241-248. ISSN: }\end{array}$ https://www.revista.ccba.uady.mx/ojs/index.php/TSA/article/view/2838

GARCÍA-GARCÍA R, López-Malo A, Palou E. 2011. Bactericidal action of binary and ternary mixtures of carvacrol, thymol, and eugenol against Listeria innocua. Journal of Food Science. 76(2):M95-M100. https://doi.org/10.1111/j.1750-3841.2010.02005.x 
KIM E, Guan L, Lee SJ, Lee SM, Lee SS, Lee ID, Lee SK, Lee SS. 2015. Effects of Flavonoid-rich Plant Extracts on In vitro Ruminal Methanogenesis, Microbial Populations and Fermentation Characteristics. Asian-Australasian. Journal of Animal Sciences. 28(4):530-537. https://doi.org/10.5713/ajas.14.0692

KURNIAWATI A, Yusiati LM, Widodo W, Artama WT. 2020. Study of Local Herb Potency as Rumen Modifier: Red Ginger (Zingiber officinale Var. Rubrum) Addition Effect on In Vitro Ruminal Nutrient Digestibility. Animal Production. 21(1):30-37. https://doi.org/10.20884/1.jap.2019.21.1.713

MOSS AR, Jouany JP, Newbold J. 2000. Methane production by ruminants: Its contribution to global warming. Annales de zootechnie. 49(3):231-253. https://doi.org/10.1051/animres:2000119

NILE SH, Nile AS, Keum YS. 2017. Total phenolics, antioxidant, antitumor, and enzyme inhibitory activity of Indian medicinal and aromatic plants extracted with different extraction methods. 3 Biotech. 7(1):76. https://doi.org/10.1007/s13205-017-0706-9

RODRÍGUEZ-GARCÍA I, Silva-Espinoza B, Ortega-Ramírez L, Leyva J, Siddiqui Md, Cruz-Valenzuela M, González-Aguilar G, Ayala-Zavala J. 2015. Oregano Essential Oil as an Antimicrobial and Antioxidant Additive in Food Products. Critical Reviews in Food Science and Nutrition. 56(10):1717-1727. https://doi.org/10.1080/10408398.2013.800832

SEJIAN V, Bhatta R, Soren NM, Malik PK, Ravindra JP, Prasad CS, Lal R. 2015. Introduction to Concepts of Climate Change Impact on Livestock and Its Adaptation and Mitigation. En: Sejian V, Gaughan J, Baumgard L, Prasad C. (eds) Climate Change Impact on Livestock: Adaptation and Mitigation. Springer, New Delhi. Pp. 1-25. ISBN: 978-81322-2265-1. https://doi.org/10.1007/978-81-322-2265-1_1

SORENTINO S, Landmesser U. 2005. Nonlipid-lowering effects of statins. Current Treatment Options Cardiovascular $\quad$ Medicine. https://doi.org/10.1007/s11936-005-0031-1

TEIXEIRA B, Marques A, Ramos C, Serrano C, Matos O, Neng N. 2013. Chemical composition and bioactivity of different oregano (Origanum vulgare) extracts and essential oil. Journal of Science of Food and Agriculture. 93:2707-2714. https://doi.org/10.1002/jsfa.6089 
THEODOROU MK, Williams BA, Dhanoa MS, McAllan AB, France J. 1994. A simple gas production method using a pressure transducer to determine the fermentation kinetics of ruminant feeds. Animal feed science and technology. 48: 185-197. https://doi.org/10.1016/0377-8401(94)90171-6

UGBOGU EA, Elghandour MM, Ikpeazu VO, Buendía GR, Molina OM, Arunsi UO, Salem AZ. 2019. The potential impacts of dietary plant natural products on the sustainable mitigation of methane emission from livestock farming. Journal of Cleaner Production. 213:915-925. https://doi.org/10.1016/j.jclepro.2018.12.233

WANG J, Liu M, Wu Y, Wang L, Liu J, Jiang L, Yu Z. 2016. Medicinal herbs as a potential strategy to decrease methane production by rumen microbiota: a systematic evaluation with a focus on Perilla frutescens seed extract. Applied Microbiology and Biotechnology. 100(22):9757-9771. https://doi.org/10.1007/s00253-016-7830-z

YUAN H, Ma Q, Ye L. y Piao G. 2016. The Traditional Medicine and Modern Medicine from Natural Products. Molecules. 21(5):559. https://doi.org/10.3390/moléculas21050559 